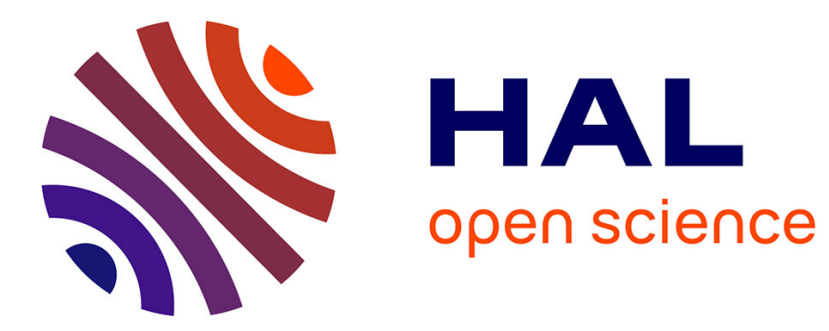

\title{
Robust sideslip angles observer for accurate off-road path tracking control
}

Roland Lenain, Mathieu Deremetz, Jean-Baptiste Braconnier, Benoit Thuilot, Vincent Rousseau

\section{To cite this version:}

Roland Lenain, Mathieu Deremetz, Jean-Baptiste Braconnier, Benoit Thuilot, Vincent Rousseau. Robust sideslip angles observer for accurate off-road path tracking control. Advanced Robotics, 2017, 31 (9), pp.453 - 467. 10.1080/01691864.2017.1280414 . hal-01658404

\author{
HAL Id: hal-01658404 \\ https://hal.uca.fr/hal-01658404
}

Submitted on 16 May 2020

HAL is a multi-disciplinary open access archive for the deposit and dissemination of scientific research documents, whether they are published or not. The documents may come from teaching and research institutions in France or abroad, or from public or private research centers.
L'archive ouverte pluridisciplinaire HAL, est destinée au dépôt et à la diffusion de documents scientifiques de niveau recherche, publiés ou non, émanant des établissements d'enseignement et de recherche français ou étrangers, des laboratoires publics ou privés. 


\title{
FULL PAPER
}

\section{Robust sideslip angles observer for accurate off-road path tracking control}

\author{
Roland Lenain $^{\mathrm{a} *}$, Mathieu Deremetz ${ }^{\mathrm{a}}$, Jean-Baptiste Braconnier ${ }^{\mathrm{a}}$, Benoit Thuilot ${ }^{\mathrm{bc}}$, Vincent Rousseau $^{\mathrm{a}}$ \\ ${ }^{a}$ Irstea, 9 avenue Blaise Pascal, CS 20085, 63172 Aubière, France \\ ${ }^{b}$ Clermont Université, Université Blaise Pascal, Institut Pascal, BP 10448, 63000 Clermont-Fd, France ${ }^{c}$ CNRS, \\ UMR 6602, Institut Pascal, 63171 Aubière, France
}

(Received 00 Month 20XX; accepted 00 Month 20XX)

\begin{abstract}
This paper proposes a control strategy to achieve high accurate path tracking in off-road conditions. The approach is based on adaptive and predictive techniques to account for sliding effects and actuator properties. An extended kinematic model is designed using sideslip angles definition. An observer is proposed to estimate on-line these variables, independently from the reference path and robot velocity. Thanks to the proposed approach, high accurate path tracking can then be achieved whatever the shape of the reference path and the task to be achieved (practical stabilization or moving object tracking).
\end{abstract}

Keywords: off-road mobile robot, path tracking, adaptive control, grip conditions, nonlinear control

\section{Introduction}

Because of its promising benefits for everyday life in different areas (transportation, agriculture, surveillance, etc.), mobile robotics has been the object of intensive research. To cover the diversity of practical applications, several problems have to be addressed, such as terrain crossing [25], posture stabilization [10] or path tracking [19]. Specifically, for the path tracking case, generic solutions have been proposed when robot motion satisfies pure rolling without sliding assumptions : they are based on the classification of kinematic models of mobile robots introduced in [4], from which various control techniques can be considered, such as exact linearization as proposed in [23]. As long as mobile robots move on terrains with high grip conditions at rather low speeds, such as in urban applications, non-sliding assumptions are realistic and the above-mentioned control approaches are satisfactory [13]. In contrast, in the more and more popular off-road applications, such as exploration [3] or agriculture [2,11], robots move on natural grounds, with non-ideal and varying grip conditions. As a result, the actual robot behavior is quite different from the one predicted by the kinematic models derived from non-sliding assumptions [8]. The tracking control laws have then to be adapted to the changing interaction between robot and environment, otherwise convergence to the reference path may no longer be ensured [14].

To meet this aim, several approaches may be considered. First, the sliding effects can be described as a perturbation acting on the classical model [1] and robust control techniques can then be designed in order to reject this perturbation, as achieved in $[7,18,20]$. These approaches propose however two difficulties : on one hand, the bounds on the sliding perturbation to be provided to the robust control laws are rather difficult to appreciate, and on the other hand these control laws often appear conservative and/or may generate oscillating behaviors. Alternatively, the sliding effects can be incorporated into extended robot models. Control laws accounting explicitly for these effects can then be designed and achieve high performances (and robust control techniques can possibly supplement these control laws to address other unmodeled perturbations).

\footnotetext{
*Corresponding author. Email : roland.lenain@irstea.fr
} 


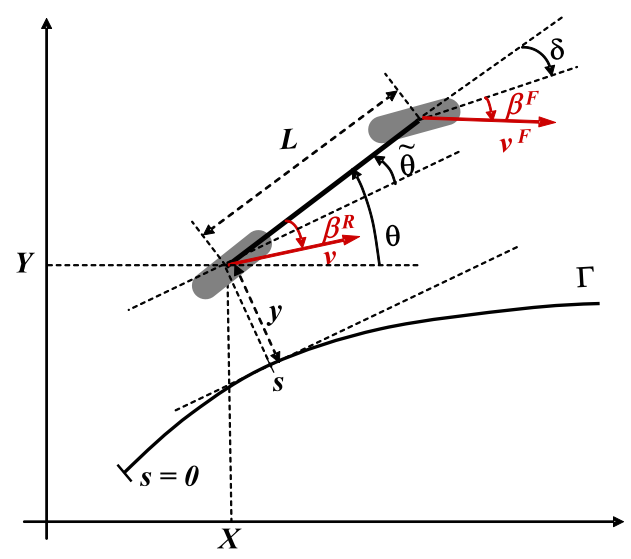

Figure 1.: Extended kinematic model

In order to tackle low grip conditions, the use of dynamical representations permits to account for their influence on robot behavior [9], but requires the knowledge of numerous parameters, difficult to be known, and which are moreover variable in uncertain environment. Alternatively, the motion description using an extended kinematic model accounting for sideslip angles, as proposed in [15] and generalized in [26], permits to account for skidding effects without introducing complex parameters. The use of extended kinematic models implies the on-line estimation of sideslip angles, which are hardly measurable directly. As achieved in previous work [17], the indirect estimation using observer theory is possible with a satisfactory accuracy. Nevertheless, these previously developed approaches suffer for several drawbacks. First, they are related to the trajectory to be followed, which may introduce noises and discontinuities. Secondly, a singularity is present when the velocity is null, which implies some switches with respect to the robot speed. To clear up the limitations of the former observer, this paper proposes a new observer, based on absolute motion (without a trajectory to be followed) and advanced observer functions allowing to be independent from the velocity. As a consequence, very general path tracking applications in off-road conditions can be achieved safely and with high accuracy.

The paper is organized as follows. First, robot modeling is addressed. In order to account for sliding effects, extended kinematic models are considered and expressed in an absolute frame and in a frame attached to the reference path. Next, sideslip angle estimation is investigated. The observer proposed in [6] is first recalled and then the new robust observer, which is the main contribution of this paper, is derived. The adaptive and predictive path tracking control law proposed in [6] is next briefly exposed. Finally, experimental results are provided to demonstrate the efficiency of the overall control strategy.

\section{Modeling}

As highlighted previously, high accurate path tracking cannot be achieved in off-road context if grip conditions are not taken into consideration. Basically, the motion control of wheeled mobile robots is based on pure kinematic models, classified in [4]. The assumption of a local even ground (all the wheels are in contact with the soil) permits to consider a reduced number of wheels. For a car-like mobile robot, the two front steering wheels are viewed as a unique tire with a unique steering angle, while the rear axle is reduced to a unique wheel. A huge literature (as it can be illustrated for instance in [24]) shows the relevance of such a modeling for robots as long as the robots do not slide. The rolling without sliding assumption gives the orientation of the speed vector at each contact point, which is no more the case when the robot moves on a natural environment. Complex models permit to consider such a phenomenon (some examples of dynamical models, considering specifically tire/ground interaction, may be found in [22] or in [5]). Nevertheless, such models require a lot of parameters, which cannot be estimated in a varying environment. Such models are then mainly dedicated to car and tire design or simulation, but are hardly 
tractable for control purpose.

Alternatively, extended kinematic models have been designed in [14] and generalized in [27] in order to account for the influence of sliding in the motion control. Specifically, the model proposed in [15] for car-like mobile robots is considered here : as for the non-sliding case (see e.g. [21]), the robot is viewed as a bicycle model with one steerable wheel for the front axle and a second one for the rear axle, see Figure 1. Nevertheless, in order for sliding effects to be taken into account, the orientation of the speed vector at wheel centers is no longer assumed to be superimposed with the tire orientation, but differs from it by time-varying angles $\beta^{F}$ and $\beta^{R}$ (for the front and rear axles) named sideslip angles.

Modeling can be carried out either in an absolute frame or in a frame attached to the path to be followed.

In the first case, a possible robot state vector consists in the 3-tuple $(X, Y, \theta)$, where $(X, Y)$ stand for the coordinates of the center of the robot rear axle in the absolute frame and $\theta$ is the robot centerline orientation with respect to that frame, see Figure 1. In addition, let $v \delta$ and $L$ denote the linear velocity at the center of the robot rear axle, the front wheel steering angle and the robot wheelbase, respectively. Relying on these notations, the equations of the extended kinematic model in the absolute frame are quite straightforward :

$$
\left\{\begin{aligned}
\dot{X} & =v \cos \left(\theta+\beta^{R}\right) \\
\dot{Y} & =v \sin \left(\theta+\beta^{R}\right) \\
\dot{\theta} & =v \cos \left(\beta^{R}\right) \frac{\tan \left(\delta+\beta^{F}\right)-\tan \left(\beta^{R}\right)}{L}
\end{aligned}\right.
$$

In the second case, the robot configuration can be described without ambiguity by the 3-tuple $(s, y, \tilde{\theta})$, where $s$ denotes the curvilinear abscissa of the center of the robot rear axle with respect to the reference path $\Gamma$ (i.e. the distance achieved by the robot along the path to be followed), when $y$ and $\tilde{\theta}$ stand for the robot lateral and angular deviations with respect to $\Gamma$, see Figure 1. Let also $c(s)$ denote the curvature of the reference path $\Gamma$ at abscissa $s$. It can then be established, see for instance [15], that the extended kinematic model expressed in the frame attached to the reference path, also named Frénet frame, is :

$$
\left\{\begin{aligned}
\dot{s} & =v \frac{\cos \left(\tilde{\theta}+\beta^{R}\right)}{1-c(s) y} \\
\dot{y} & =v \sin \left(\tilde{\theta}+\beta^{R}\right) \\
\dot{\tilde{\theta}} & =v\left[\cos \left(\beta^{R}\right) \frac{\tan \left(\delta+\beta^{F}\right)-\tan \left(\beta^{R}\right)}{L}-\ldots\right. \\
\ldots & \left.\ldots \frac{c(s) \cos \left(\tilde{\theta}+\beta^{R}\right)}{1-c(s) y}\right]
\end{aligned}\right.
$$

The model singularity $1-c(s) y=0$ is never encountered in practice when the path tracking is properly initialized : the robot is never located at the curvature center of the reference path. The five classes of kinematic models introduced in [4] to describe the motion of any mobile robot in the non-sliding case can all be extended in the same way when sliding effects have to be taken into account, see for instance [26], and the exact linearizing properties of chained systems can then be applied. As a consequence, if the values of the sideslip angles $\beta^{F}$ and $\beta^{R}$ can be supplied online (see next section), the numerous control approaches developed for mobile robots in the non-sliding case remains straightforwardly applicable.

For the path tracking problem addressed here for the car-like mobile robot, model (2) appears more convenient than model (1), since the control objective can be expressed directly in terms of the state variables : the control law should ensure the convergence of $y$ and $\tilde{\theta}$ with zero. Consequently, control design in section 4 is carried out from model (2). In contrast, for the online estimation of the sideslip angles, model (2) is this time far less attractive : since state variables are defined with respect to the reference path, any perturbation in the reference path (noisy values or discontinuities) leads to inconsistent changes in the state variable values that, if reported into equations (2), could be "explained" only by large sideslip angle values. In other words, perturbations in the reference path would be misinterpreted as large sliding and this may 
seriously damage control law performances. As a consequence, in order for sideslip angle estimation to be insensitive to reference path imperfections, it appears preferable to design sliding observer from model (1), as detailed in the next section.

Such extended kinematic models are devoted to control purpose. As a result, they do not aim at describing accurately all the robot dynamics. The objective is to determine the influence of control variables on the general robot motion. The inaccuracies are neglected and basically expected to be compensated by the adaptive control strategy hereafter described.

\section{Sideslip angle estimation}

The sideslip angle observer proposed in this paper is detailed in section 3.2. Beforehand, since the capabilities of this new observer designed from model (1) are compared in section 5 with those of a previous observer developed from model (2), this former observer is briefly recalled in section 3.1. The objective is to estimate the sideslip angles $\beta^{F}$ and $\beta^{R}$ representative of the influence of sliding on the robot's behavior, without adding sensors.

\subsection{Previous approach}

The path tracking control law to be designed in section 4 from model (2) should be supplied online with relevant values for the sideslip angles $\beta^{F}$ and $\beta^{R}$. Since these two variables cannot be easily measured, an indirect measurement should be proposed. In [6], an observer based also on model (2) has been developed using the duality principle between observation and control. More precisely, in the sequel the 2-dimensional vector $\zeta=[y \quad \tilde{\theta}]^{T}$ gathers the actual lateral and angular deviations supplied by the robot sensors and $\hat{\zeta}=\left[\begin{array}{ll}\hat{y} & \hat{\tilde{\theta}}\end{array}\right]^{T}$ is an estimation of this vector evaluated from the following state-space model :

$$
\dot{\hat{\zeta}}=f_{F}(\hat{\zeta}, \delta, 0)+B(\hat{\zeta}, \delta) u
$$

The virtual control input of this model is the estimation to be determined of the two sideslip angles, i.e. $u=\left[\hat{\beta}^{F} \hat{\beta}^{R}\right]^{T}$, the function $f_{F}(\hat{\zeta}, \delta, u)$ is the right-hand side terms of the two last equations in model (2) where the actual values $y, \tilde{\theta}, \beta^{F}$ and $\beta^{R}$ have been replaced by the estimated ones $\hat{\zeta}$ and $u$, and eventually $B(\hat{\zeta}, \delta)$ is the partial derivative of $f_{F}$ with respect to $u$ evaluated at $u=\left[\begin{array}{ll}0 & 0\end{array}\right]^{T}: B(\hat{\zeta}, \delta)=\frac{\partial f_{F}}{\partial u}(\hat{\zeta}, \delta, 0)$. State-space model (3) can then be understood as the Taylor series expansion of the two last equations in model (2) in the variable $u$ up to the first order and evaluated around $u=\left[\begin{array}{ll}0 & 0\end{array}\right]^{T}$. The expression of $B(\hat{\zeta}, \delta)$ can be easily computed from (2):

$$
B(\hat{\zeta}, \delta)=\left[\begin{array}{cc}
0 & v \cos (\hat{\tilde{\theta}}) \\
\frac{v}{L \cos ^{2} \delta} & v \frac{c(s) \sin (\hat{\tilde{\theta}})}{1-c(s) \hat{y}}-\frac{v}{L}
\end{array}\right]
$$

This matrix is clearly invertible provided that $\hat{\tilde{\theta}} \neq \frac{\pi}{2}[\pi]$ and $v \neq 0$. If the robot is properly initialized (i.e. if its initial deviations with respect to the reference path are not very large), the first condition is satisfied. In addition, the sideslip angle estimation will be frozen at low speeds so that the second condition may be satisfied as well. Next, since $B(\hat{\zeta}, \delta)$ is invertible, exponential convergence of the estimated state $\hat{\zeta}$ with the measured one $\zeta$ can be easily imposed by computing the estimated sideslip angles $u$ as follows :

$$
u=B(\hat{\zeta}, \delta)^{-1}\left(G e-f_{F}(\hat{\zeta}, \delta, 0)+\dot{\zeta}\right)
$$


where $e=\hat{\zeta}-\zeta$ and $G$ is a $2 \times 2$ Hurwitz matrix introduced for convergence rate tuning (reporting (5) into (3) leads to $\dot{e}=G e$ ). More details can be found in [6]. The convergence of $e$ with zero means that model (3) with control law (5) is representative of the robot actual behavior, and consequently the sideslip angles supplied by (5) can be considered as representative of actual sliding conditions. Adaptation law (5) is therefore a first sliding observer, to be compared in section 5 to the new observer proposed below.

\subsection{New observer}

The previous observation strategy appears natural, since the same model (2) is used for control and estimation. Nevertheless, it requires the inversion of matrix $B(\hat{\zeta}, \delta)$ defined by (4), which is impossible when the robot velocity $v$ approaches zero, and moreover all the state variables are defined with respect to the reference path, so that this observer is sensitive to any imperfection in the reference path, as detailed at the end of section 2. Consequently, an alternative observation approach is proposed here relying on model (1) expressed in an absolute frame. Moreover, a different observation strategy is used, so that the observer does no longer need to be frozen when the robot moves slowly or stops.

\subsubsection{Notation}

In order to design the proposed observer, the non-linear model (1) is first rewritten as follows :

$$
\dot{\xi}=\left[\begin{array}{c}
\dot{\xi}_{\text {pos }} \\
\dot{\xi}_{\beta}
\end{array}\right]=\left[\begin{array}{c}
f(\xi, v, \delta) \\
0_{2 \times 1}
\end{array}\right]
$$

where the 5-dimensional state vector $\xi$ is constituted of $\xi_{\text {pos }}=\left[\begin{array}{lll}X & Y & \theta\end{array}\right]^{T}$, which represents the absolute robot posture (position and orientation) supposed to be measured, and $\xi_{\beta}=\left[\begin{array}{ll}\beta^{F} & \beta^{R}\end{array}\right]^{T}$, which gathers the two sideslip angles to be estimated. The function $f(\xi, v, \delta)$ is directly the right-hand side terms of model (1) :

$$
f(\xi, \delta, v)=\left[\begin{array}{c}
v \cos \left(\theta+\beta^{R}\right) \\
v \sin \left(\theta+\beta^{R}\right) \\
v \cos \left(\beta^{R}\right) \frac{\tan \left(\delta+\beta^{F}\right)-\tan \left(\beta^{R}\right)}{L}
\end{array}\right]
$$

and the derivative of sideslip angles is here considered null. Expressions for the derivative of the sideslip angles could have been obtained from dynamical equations of motion, see for instance [22], but in that case numerous parameters badly known or largely varying would have been mandatory, see [17]. In this paper, an extended kinematic modeling has been preferred in order to avoid such a difficulty. So, to be consistent with this choice, the derivative of sideslip angles is here neglected and the short observer settling time (via gain tuning) is supposed to compensate for the lack of knowledge in sideslip angle dynamics. This is completely satisfactory as long as the mobile robot moves at moderate speed, as shown in section 5 .

\subsubsection{Observer equations}

From state space model (6), the following observer is proposed :

$$
\dot{\hat{\xi}}=\left[\begin{array}{c}
\dot{\hat{\xi}}_{p o s} \\
\dot{\hat{\xi}}_{\beta}
\end{array}\right]=\left[\begin{array}{c}
f\left(\xi_{\text {pos }}, \hat{\xi}_{\beta}, \delta, v\right)+\alpha_{\text {pos }} \\
\alpha_{\beta}
\end{array}\right]
$$

where $\hat{\xi}=\left[\begin{array}{ll}\hat{\xi}_{\text {pos }} & \hat{\xi}_{\beta}\end{array}\right]^{T}$ is the observed state and $\alpha_{\text {pos }}$ and $\alpha_{\beta}$ are 3-dimensional and 2-dimensional functions, respectively. Next, the objective is to design these two functions so that the observation error $\tilde{\xi}=\xi-\hat{\xi}$ 
tends to zero. Since $\xi_{\text {pos }}$ is assumed to be measured, the observed state $\hat{\xi}_{\text {pos }}$ does not matter, the main goal here is to find a relevant estimation $\hat{\xi}_{\beta}$ of $\xi_{\beta}$. It is shown below that this objective can be met with the two following functions :

$$
\left\{\begin{aligned}
\alpha_{\text {pos }} & =K_{\text {pos }} \tilde{\xi}_{\text {pos }} \\
\alpha_{\beta} & =K_{\beta}\left[\frac{\partial f}{\xi_{\beta}}\left(\xi_{\text {pos }}, \hat{\xi}_{\beta}, \delta, v\right)\right]^{T} \tilde{\xi}_{\text {pos }}
\end{aligned}\right.
$$

where $\tilde{\xi}_{\text {pos }}=\xi_{\text {pos }}-\hat{\xi}_{\text {pos }}$ is the observation error related to robot posture, which can be measured, $K_{\text {pos }}$ is a diagonal positive $3 \times 3$ matrix and $K_{\beta}$ is a positive scalar.

\subsubsection{Sketch of proof}

Let us consider the Lyapunov candidate function $V$ defined by :

$$
V=V_{1}+V_{2}
$$

with :

$$
\left\{\begin{aligned}
V_{1} & =\frac{1}{2} \tilde{\xi}_{\text {pos }}^{T} K_{\beta} \tilde{\xi}_{\text {pos }} \\
V_{2} & =\frac{1}{2} \tilde{\xi}_{\beta}^{T} \tilde{\xi}_{\beta}
\end{aligned}\right.
$$

Functions $V_{1}$ and $V_{2}$ (and consequently $V$ ) are clearly positive and their time derivatives are :

$$
\left\{\begin{array}{l}
\dot{V}_{1}=K_{\beta} \dot{\tilde{\xi}}_{\text {pos }}^{T} \tilde{\xi}_{\text {pos }} \\
\dot{V}_{2}=\tilde{\xi}_{\beta}^{T} \dot{\tilde{\xi}}_{\beta}
\end{array}\right.
$$

From (6) and (8), it can also be computed that :

$$
\left\{\begin{aligned}
\dot{\tilde{\xi}}_{p o s} & =f\left(\xi_{\text {pos }}, \xi_{\beta}, \delta, v\right)-f\left(\xi_{\text {pos }}, \hat{\xi}_{\beta}, \delta, v\right)-\alpha_{\text {pos }} \\
\dot{\tilde{\xi}}_{\beta} & =\dot{\xi}_{\beta}-\alpha_{\beta}
\end{aligned}\right.
$$

At this step, the two following assumptions are introduced :

1. The observation error on sideslip angles (i.e. $\tilde{\xi}_{\beta}$ ) is small enough so that a Taylor series expansion of function $f\left(\xi_{\text {pos }}, \hat{\xi}_{\beta}, \delta, v\right)$ around $\hat{\xi}_{\beta}$ can be considered :

$$
f\left(\xi_{\text {pos }}, \xi_{\beta}, \delta, v\right)=f\left(\xi_{\text {pos }}, \hat{\xi}_{\beta}, \delta, v\right)+\frac{\partial f}{\xi_{\beta}}\left(\xi_{\text {pos }}, \hat{\xi}_{\beta}, \delta, v\right) \tilde{\xi}_{\beta}
$$

2. The time derivative of actual sideslip angles $\dot{\xi}_{\beta}$ is negligible, or at least compensated by the corresponding observer dynamics $\dot{\hat{\xi}}_{\beta}$ imposed by $\alpha_{\beta}$.

Considering these assumptions in equations (13) and replacing also $\alpha_{\text {pos }}$ and $\alpha_{\beta}$ by their expressions (9) lead to :

$$
\left\{\begin{array}{c}
\dot{\tilde{\xi}}_{\text {pos }}=\frac{\partial f}{\xi_{\beta}}\left(\xi_{\text {pos }}, \hat{\xi}_{\beta}, \delta, v\right) \tilde{\xi}_{\beta}-K_{\text {pos }} \tilde{\xi}_{\text {pos }} \\
\dot{\tilde{\xi}}_{\beta}=-K_{\beta}\left[\frac{\partial f}{\xi_{\beta}}\left(\xi_{\text {pos }}, \hat{\xi}_{\beta}, \delta, v\right)\right]^{T} \tilde{\xi}_{\text {pos }}
\end{array}\right.
$$


Injecting now these expressions into the time derivative of the Lyapunov functions shown in (12) ensures that :

$$
\left\{\begin{aligned}
\dot{V}_{1}= & -K_{\beta} \tilde{\xi}_{\text {pos }}^{T} K_{\text {pos }} \tilde{\xi}_{\text {pos }}+\ldots \\
& \ldots K_{\beta} \tilde{\xi}_{\beta}^{T}\left[\frac{\partial f}{\xi_{\beta}}\left(\xi_{\text {pos }}, \hat{\xi}_{\beta}, \delta, v\right)\right]^{T} \tilde{\xi}_{p o s} \\
\dot{V}_{2}= & -K_{\beta} \tilde{\xi}_{\beta}^{T}\left[\frac{\partial f}{\xi_{\beta}}\left(\xi_{\text {pos }}, \hat{\xi}_{\beta}, \delta, v\right)\right]^{T} \tilde{\xi}_{p o s}
\end{aligned}\right.
$$

and, in view of (10), the time derivative of the complete Lyapunov function is :

$$
\dot{V}=-K_{\beta} \tilde{\xi}_{\text {pos }}^{T} K_{\text {pos }} \tilde{\xi}_{\text {pos }}
$$

Since $K_{\beta}$ is a positive scalar and $K_{\text {pos }}$ is a positive definite diagonal matrix, $\dot{V}$ is negative and equal to zero if and only if $\tilde{\xi}_{\text {pos }}=0$. It then follows that $\tilde{\xi}_{\text {pos }}$ converges with zero, i.e. the observed position and orientation converge with their corresponding measure. Next, in order to investigate the behavior of $\tilde{\xi}_{\beta}$, let us inject $\tilde{\xi}_{\text {pos }}=0$ into the observer error dynamic (14). It can be established that :

$$
\frac{\partial f}{\xi_{\beta}}\left(\xi_{p o s}, \hat{\xi}_{\beta}, \delta, v\right) \tilde{\xi}_{\beta} \rightarrow 0
$$

Computations show that the partial derivative of $f$ with respect to $\xi_{\beta}$ is null only when the velocity $v$ is null and is not singular provided that $\theta+\hat{\beta}^{R} \neq \frac{\pi}{2}[\pi]$ and $\delta+\hat{\beta}^{F} \neq \frac{\pi}{2}[\pi]$, both conditions that are satisfied in practice. As a consequence, when the robot is moving, the observer (8) with the chosen functions (9) ensures that the overall observation error tends to zero $: \tilde{\xi}=0$. In particular, this demonstrates that the estimated sideslip angles converge with the actual (but unmeasured) ones : $\hat{\xi}_{\beta} \rightarrow \xi_{\beta}$. As a result, a relevant estimation of sideslip angles $\left(\hat{\beta}^{F}, \hat{\beta}^{R}\right)$ is available and representative of the actual robot motion.

\section{Control law}

\subsection{Adaptive control law design}

The two observers presented above can supply online relevant estimations of the sideslip angles $\hat{\beta}^{F}$ and $\hat{\beta}^{R}$. As a result, all the variables in model (2) are available and this model can then be used to address path tracking.

Since the state variables of model (2) are defined with respect to the reference path, the control objective can be expressed in a very simple way : $y$ and $\tilde{\theta}$ should converge with zero, whatever the velocity $v$. In the non-sliding case, it has been shown in [23] that this objective can be achieved by turning the non-linear model (2) (with $\beta^{F}=\beta^{R}=0$ ) into a so-called chained form by means of state and control transformations, in order to rely eventually on standard linear control techniques. Since the structure of extended kinematic models is unchanged with respect to the kinematic models derived in the non-sliding case, this control approach can still be considered here. Specifically, the state and control transformations (18) and (19) permit to convert the extended kinematic model (2) into the chained form (20) :

$$
\begin{gathered}
{[s, y, \tilde{\theta}] \rightarrow\left[a_{1}, a_{2}, a_{3}\right]=\left[s, y,(1-c(s) y) \tan \left(\tilde{\theta}+\beta^{R}\right)\right]} \\
{[v, \delta] \rightarrow\left[m_{1}, m_{2}\right]=\left[\frac{v \cos \left(\tilde{\theta}+\beta^{R}\right)}{1-c(s) y}, \frac{d a_{3}}{d t}\right]}
\end{gathered}
$$




$$
\left\{\begin{array}{l}
\dot{a}_{1}=\frac{d a_{1}}{d t}=m_{1} \\
\dot{a}_{2}=\frac{d a_{2}}{d t}=a_{3} m_{1} \\
\dot{a}_{3}=\frac{d a_{3}}{d t}=m_{2}
\end{array}\right.
$$

Next, since a closed loop behavior independent from the velocity is desired, the derivative with respect to time is replaced by a derivation with respect to the curvilinear abscissa $s=a_{1}$. More precisely, by introducing the notation $a_{i}^{\prime}=\frac{d a_{i}}{d a_{1}}$, system (20) can be rewritten as :

$$
\left\{\begin{array}{l}
a_{1}^{\prime}=1 \\
a_{2}^{\prime}=a_{3} \\
a_{3}^{\prime}=m_{3}
\end{array}\right.
$$

where $m_{3}=\frac{m_{2}}{m_{1}}$ is a new virtual control variable related to the actual control variable $\delta$ according to (18) and (19). Convergence of the robot lateral deviation $y$ with zero can then be ensured by choosing the following expression for the virtual control variable $m_{3}$ :

$$
m_{3}=\frac{m_{2}}{m_{1}}=-K_{d} a_{3}-K_{p} a_{2} \quad\left(K_{d}, K_{p}>0\right)
$$

since it leads to the following $2^{\text {nd }}$ order differential equation :

$$
y^{\prime \prime}+K_{d} y^{\prime}+K_{p} y=0
$$

The settling distance (instead of settling time) can be tuned via the two positive gains $K_{p}$ and $K_{d}$, homogeneous to proportional and differential actions.

Eventually, the steering angle $\delta$ to be applied may be deduced from (22) by inverting transformations (18) and (19). The final expression for the adaptive steering law is :

$$
\begin{aligned}
\delta= & \arctan \left(\tan \left(\hat{\beta}^{R}\right)+\ldots\right. \\
& \left.\frac{L}{\cos \left(\hat{\beta}^{R}\right)}\left[\frac{c(s) \cos \tilde{\theta}_{1}}{\alpha}+\frac{A \cos ^{3} \tilde{\theta}_{1}}{\alpha^{2}}\right]\right)-\hat{\beta}^{F}
\end{aligned}
$$

with

$$
\left\{\begin{aligned}
\tilde{\theta}_{1} & =\tilde{\theta}+\hat{\beta}^{R} \\
\alpha & =1-c(s) y \\
A & =-K_{p} y-K_{d} \alpha \tan \tilde{\theta}_{1}+c(s) \alpha \tan ^{2} \tilde{\theta}_{1}
\end{aligned}\right.
$$

Control law (24) constitutes an adaptive expression since $\hat{\beta}^{F}$ and $\hat{\beta}^{R}$ are computed with one of the observers presented in section 3. Convergence of the tracking error is then ensured whatever the grip conditions, without adding any new measurement with respect to the standard equipment of a mobile robot. Nevertheless, if this reactive strategy permits a high accurate path tracking in steady state conditions (i.e. when the reference path curvature is constant or this path consists in a straight line on a slope), overshoots can still be noticed at transitions in the reference path curvature, due to the neglected dynamics of the actuator (settling time, delays, etc.). To address specifically this point, the adaptive steering law (24) is supplemented below with predictive functional control techniques. 


\subsection{Predictive curvature servoing}

In order to prevent overshoots at curvature transition phases, a predictive curvature servoing is introduced. More precisely, it has been shown in [6] that the reactive control expression (24) may be split into two terms :

$$
\delta=\delta_{\text {Traj }}+\delta_{\text {Deviation }}
$$

where $\delta_{\text {Deviation }}$ is null when sideslip angles $\beta^{F}, \beta^{R}$ and deviations $y, \tilde{\theta}$ are all null, while $\delta_{\text {Traj }}$ remains non-null in these conditions as soon as the reference path curvature $c(s)$ is non-zero. This means that the two terms play different roles : $\delta_{\text {Deviation }}$ mainly permits to compensate for sliding and unexpected errors, while $\delta_{T r a j}$ aims principally at steering the robot with respect to the reference path curvature.

Since grip conditions and unexpected errors are unpredictable by definition, $\delta_{\text {Deviation }}$ is left unchanged in the sequel. Only the term $\delta_{\text {Traj }}$ is substituted with a predictive term depending on the future curvature of the reference path $c\left(s_{H}\right)$, where $s_{H}$ is the curvilinear abscissa that should be reached after an horizon of prediction $t_{H}$ (i.e. $\left.s_{H}=s(t)+\dot{s}(t) t_{H}\right)$. More precisely, from the future curvature $c\left(s_{H}\right)$, a set point $\delta^{O b j}$

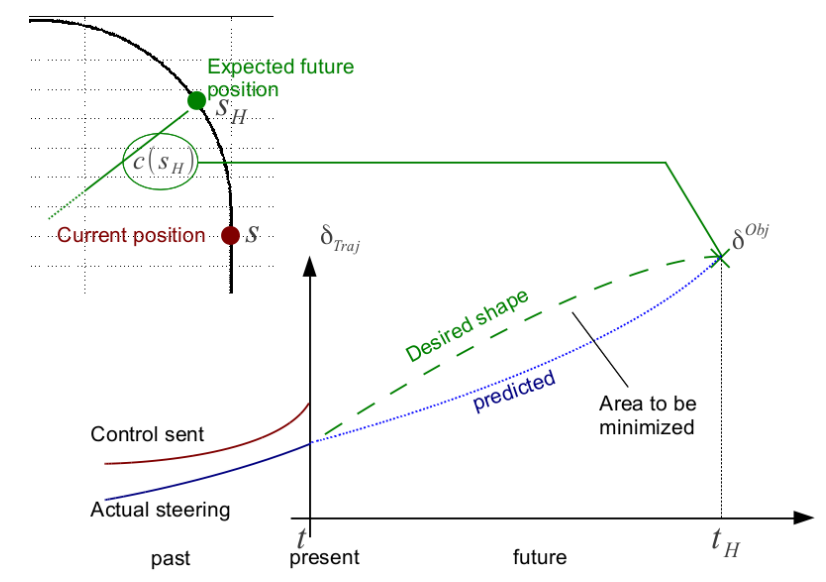

Figure 2.: Predictive curvature servoing illustration

to be reached by $\delta_{T r a j}$ after an horizon of prediction $t_{H}$ is computed, as well as a desired shape to join this objective (as illustrated in Figure 2). Next, a model for the actuator dynamics is used in order to compute the optimal sequence of control values from $t$ to $t+t_{H}$ that minimizes the error between the desired shape and the predicted values for $\delta_{T r a j}$ (so called predicted and shown in blue dotted line in Figure 2). The optimal sequence thus obtained permits to reach at best the set point imposed by the future curvature $c\left(s_{H}\right)$. Finally, the first term of this optimal sequence is named $\delta_{\text {Traj }}^{\text {Pred }}$ and is substituted with $\delta_{\text {Traj }}$ in control law (24). More details can be found in [16]. The final adaptive and predictive steering law is then :

$$
\delta_{F}=\delta_{\text {Traj }}^{\text {Pred }}+\delta_{\text {Deviation }}
$$

With control law (27), the front wheel starts to be steered before the robot enters into the curve, so that transient overshoots at curvature transition phases can be significantly reduced. As a result, the proposed control law permits to preserve a high accuracy, despite non-ideal grip conditions and fast variations in reference path curvature.

\section{Path tracking results}

The proposed adaptive control law permits to account for sliding effects despite the numerous perturbations encountered when moving on natural ground : sliding, noises or vibrations. The observation approach 
allows to estimate and integrate explicitly the effects of bad grip conditions by means of the sideslip angles. As a result, it permits to face such dynamics and to account for the nonlinear behavior of tire and variations in tire/soil interaction, without using complex dynamic models. The obtained tracking accuracy and the robustness with respect to changing conditions are illustrated by means of full scale experiments achieved in different conditions : soil geometry, grip conditions and velocity.

\subsection{Experimental setup}

The electric vehicle used for the experimentations is shown in Figure 3. Designed for all-terrain mobility, it can climb slopes up to $45^{\circ}$. Its main parameters are listed in Table 1 .

$$
\begin{aligned}
\text { Total mass : } & m=350 \mathrm{~kg} \\
\text { Yaw inertia : } & I_{z}=270 \mathrm{~kg} \mathrm{~m}^{2} \\
\text { Wheelbase : } & L=1.2 \mathrm{~m} \\
\text { Rear half-wheelbase : } & b=0.58 \mathrm{~m}
\end{aligned}
$$

Table 1.: Experimental mobile robot parameters

The main exteroceptive sensor on-board is a "Magellan ProFlex 500" RTK-GPS receiver, which supplies absolute position measurements with an accuracy of $2 \mathrm{~cm}$ at a $10 \mathrm{~Hz}$ sampling frequency, thanks to a reference station providing corrections to the moving GPS antenna. This moving antenna is located vertically above the center of the rear axle, so that the absolute position of the point to be controlled is straightforwardly obtained from the sensor.

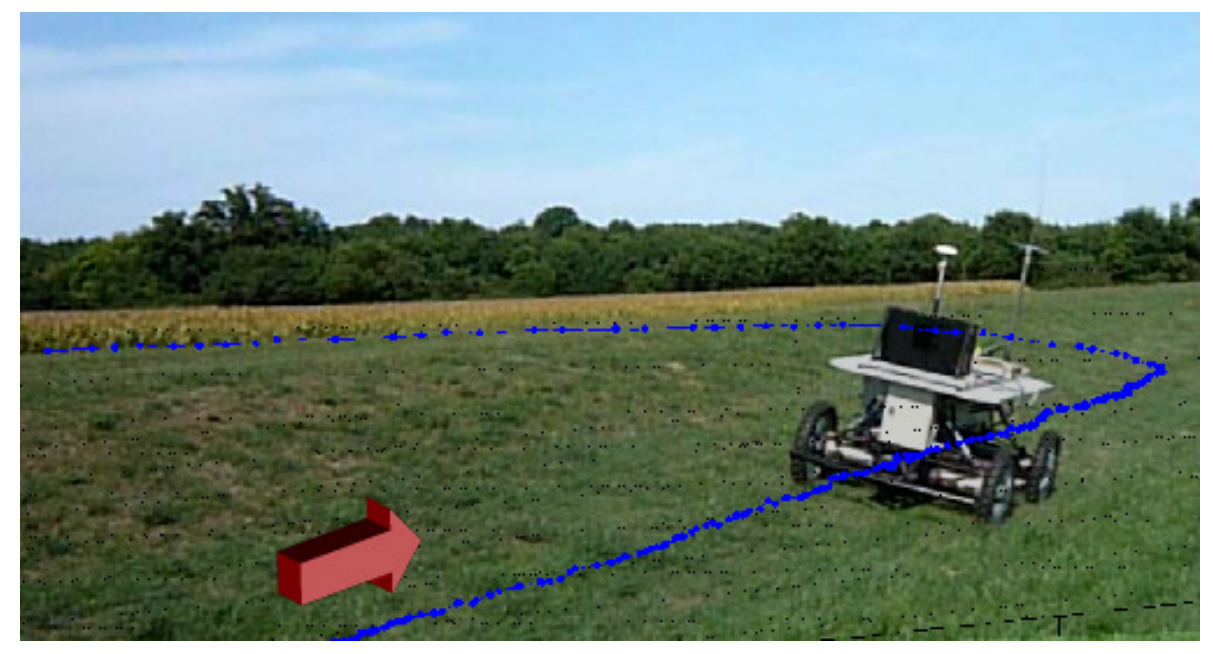

Figure 3.: Experimental platform in actual conditions

\subsection{Test without discontinuity in the path to be followed}

As it can be seen in Figure 3, the reference path (shown with blue points) has been recorded on an uneven terrain. It consists in a straight line on a sloping area with a $15^{\circ}$ lateral inclination, followed by a half-turn that ends on a flat ground and finally another straight line and another curve both on the flat part of the terrain. The entire reference path is also shown in a top 2D view in Figure 4. The robot moves on a wet grass ground generating a drift in the sloping part or when the robot turns.

First of all, the case of a reference path with no discontinuity has been considered. This path is depicted in black line in the left part in Figure 4 and the tracking errors recorded when sliding is not taken into account 
(i.e. $\left.\left(\hat{\beta}^{F}, \hat{\beta}^{R}\right)=(0,0)\right)$ and when the estimated sideslip angles delivered by the new observer (8) are used, are reported in blue plain line and green dashed-dotted line respectively in the right part in Figure 4 . Both tracking have been achieved at $3 \mathrm{~m} . \mathrm{s}^{-1}$.

Except the punctual overshoot occurring when the robot comes from the sloping area to the even ground (due to transient inconsistencies in robot localization, since the inclination of the GPS antenna located $1.6 \mathrm{~m}$ above the robot changes abruptly), the tracking error when estimated sideslip angles are reported into control law (27) is always close to zero. This very satisfactory accuracy is achieved despite the ground inclination and bad grip conditions (terrain covered in grass).

In contrast, when sliding is not taken into account, large tracking errors are recorded, depending on the robot motion and the terrain properties : $0.6 \mathrm{~m}$ in the sloping area (from curvilinear abscissa 40 to $60 \mathrm{~m}$ ), $0.8 \mathrm{~m}$ when the robot executes the half-turn $($ at $80 \mathrm{~m}$ ) and punctual errors during the second curve (from $0.3 \mathrm{~m}$ (at $110 \mathrm{~m}$ ) to $0.6 \mathrm{~m}$ (at $130 \mathrm{~m}$ )). These experimental results clearly show the importance of taking sliding into account and the relevance of the adaptive control strategy proposed in this paper to address such applications.

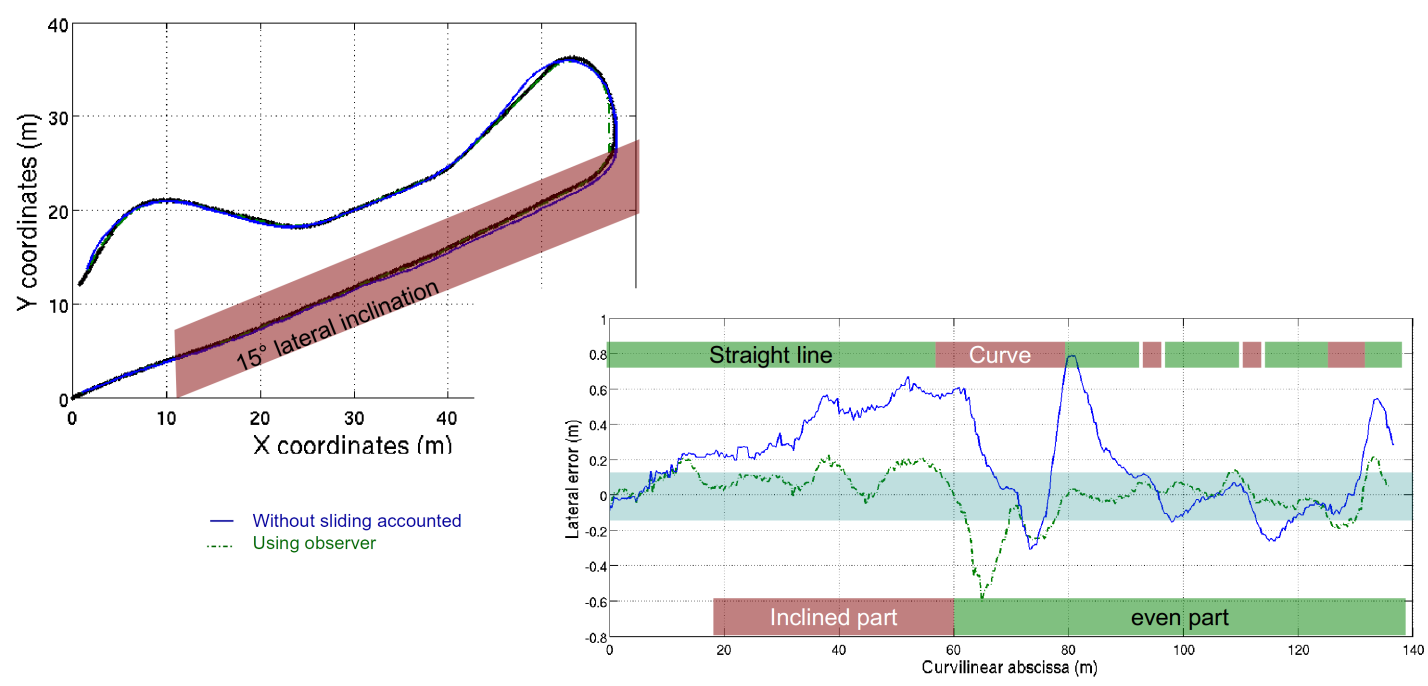

Figure 4.: Experimentation without discontinuity

\subsection{Test with a reference path presenting a discontinuity}

In order to investigate the robustness of the new observer (8), a discontinuity (i.e. a 1 m-step) has been introduced in the reference path, at the middle of the first straight line achieved on the $15^{\circ}$ sloping area. This modified reference path is shown in black plain line in Figure 5.

This reference path has been tracked three times at $3 \mathrm{~m} . \mathrm{s}^{-1}$ : without sliding accounted (depicted in blue plain line in the sequel), next with estimated sideslip angles supplied by the previous observer (5) (depicted in red dotted line), and finally with estimated sideslip angles delivered by the new observer (8) (depicted in green dashed line).

The second experimentation (with observer (5)) has been stopped just after crossing the discontinuity, since path tracking had become instable and the robot was moving apart. Once more, since this observer relies on state variables defined with respect to the reference path, the discontinuity in the reference path induces an abrupt change in these state variables that is misinterpreted as large sliding. The estimated sideslip angles delivered by this observer are then abnormally large and when reported into steering law (27) lead to path tracking instability.

In order that path tracking could be achieved with this observer, the second experimentation has been restarted, but the estimated sideslip angles supplied by observer (5) have been low-pass filtered. Path tra- 


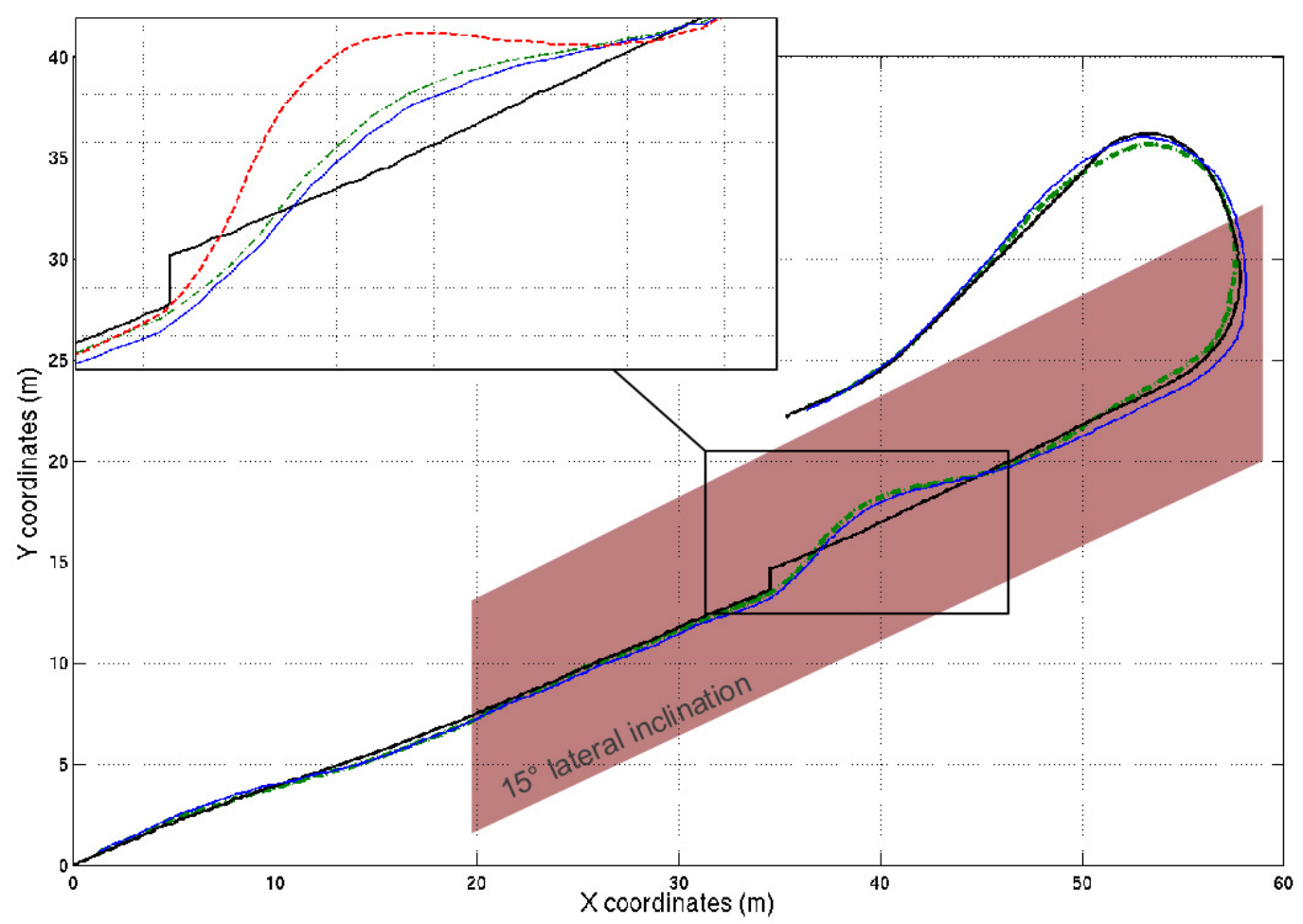

Figure 5.: Reference trajectory and tracking results

cking has been successful this time, but the performances are of course depreciated. For readability reasons, only the robot trajectories recorded in the first and third experimentations are reported in the main plot in Figure 5. The trajectory recorded in the second experimentation (with the low-pass filter) is only shown in red dashed line in the zoom on the discontinuity proposed in this figure and an important tracking error can be noticed.

In contrast, the new observer (8) is not seriously affected by the reference path discontinuity : the tracking errors reported in Figure 6 show that the overshoot due to the discontinuity is limited to $-1 \mathrm{~m}$ (at curvilinear abscissa $45 \mathrm{~m}$ ), against more than $2.5 \mathrm{~m}$ with the previous observer (5) coupled with the low-pass filter. An overshoot is here present since the robot moves on a sloping area and consequently sliding effects are mainly generated by the slope rather than by the steering action. This is also the reason why the overshoot recorded with observer (8) is similar to the one obtained when sliding is not taken into account. Nevertheless, except at the discontinuity crossing, the conventional control strategy is not satisfactory : since the robot moves on a grassy sloping ground, the lateral inclination generates an important drift and, since it is not compensated within the steering law, the tracking errors are larger $(0.3 \mathrm{~m}$ at curvilinear abscissa $35 \mathrm{~m}$ and $0.6 \mathrm{~m}$ at $62 \mathrm{~m})$ than in the third experimentation when the estimated sideslip angles delivered by observer (8) are used.

During the first experimentation, when sliding is not taken into account, the two sideslip angle observers have been run simultaneously (although they were not used) and their estimations have been recorded. The estimated front sideslip angles delivered by the two approaches are compared in Figure 7. Except at discontinuity crossing, it can be noticed that the two observers supply similar sideslip angle estimations. Consequently the same high tracking accuracy can be expected when any of these two estimations is reported into control law (27), as corroborated in Figure 6. In contrast, despite the low-pass filter, the estimated sideslip angle values supplied by the previous observer (5) at the discontinuity crossing are still larger than the ones delivered by the new observer (8) $\left(5.5^{\circ}\right.$ at curvilinear abscissa $40 \mathrm{~m}$, against $\left.3^{\circ}\right)$. Since these are experimental results, no ground truth is available for sideslip angles. Nevertheless, the large $2.5 \mathrm{~m}$ overshoot recorded on tracking error with observer (5) (against a 1 m overshoot when observer (8) is used) suggests that the values delivered by observer (8) are more relevant in this situation. As a result, observer (8) appears 


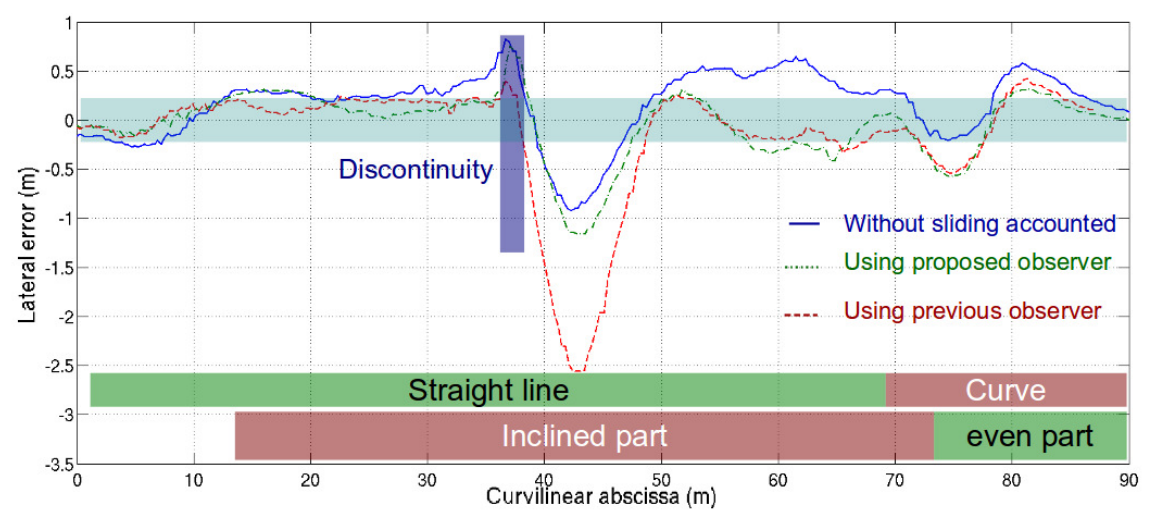

Figure 6.: Comparison of actual tracking errors

definitely more attractive since high accuracy path tracking can be preserved, even when the reference path is not perfectly continuous.

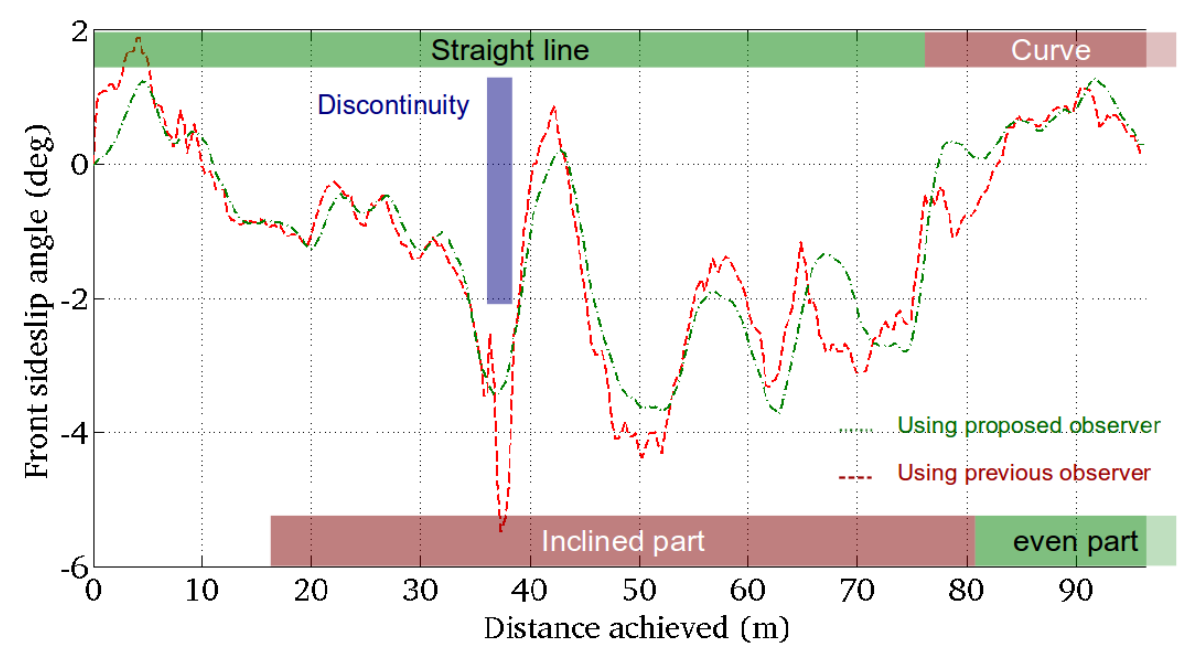

Figure 7.: Comparison of sideslip angles estimations during the first experiment

\subsection{Additional results in different conditions}

In order to test the performances and the robustness of the proposed observer, a second trajectory with harsh curvature transitions has been considered. This trajectory is depicted in Figure 8. It consists of a straight line followed by two curves and finally another straight line. The first curve has an almost constant curvature, while the curvature of the second one is increasing during the turn. In addition, the first curve is to the left, when the second one is to the right and presents at its end an S-shape which generates high sideslip angles. This reference path is achieved on different surfaces, enabling to test the proposed algorithm with respect to different grip conditions. As it can be seen in the center image in Figure 8, the trajectory starts on asphalt and next alternates between grass and asphalt. The ground is flat and the experimental testbed remains unchanged (same vehicle, same sensors). 

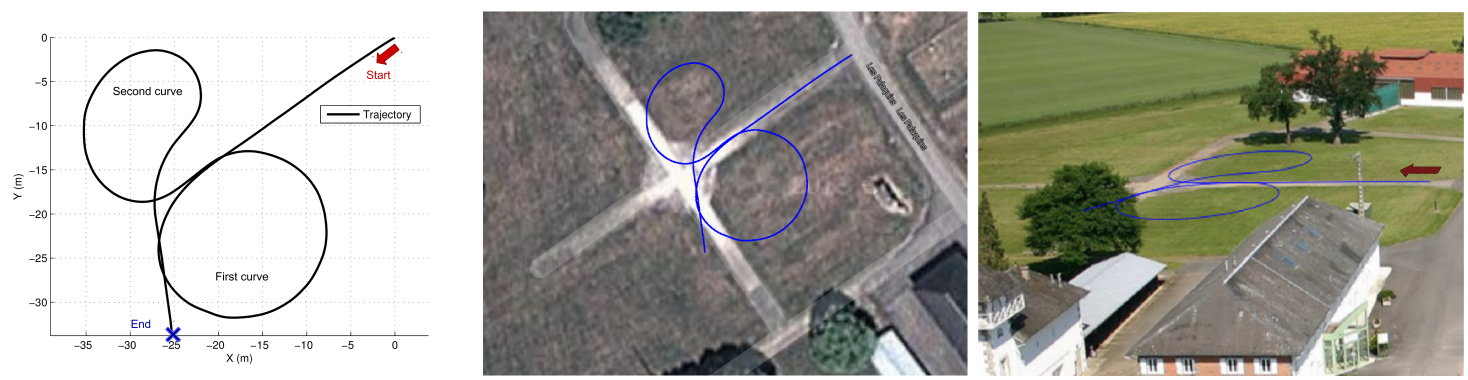

Figure 8.: Reference path

\subsubsection{Comparison of observers performances}

The results presented below were obtained by performing the tracking in three different ways (without, with previous and new observer) at two selected speeds $\left(2 \mathrm{~m} \cdot \mathrm{s}^{-1}\right.$ and $\left.4 \mathrm{~m} \cdot \mathrm{s}^{-1}\right)$. In all following figures, curves in black, red and blue correspond to the trials without observer, with the previous approach and with the one proposed in this paper, respectively.

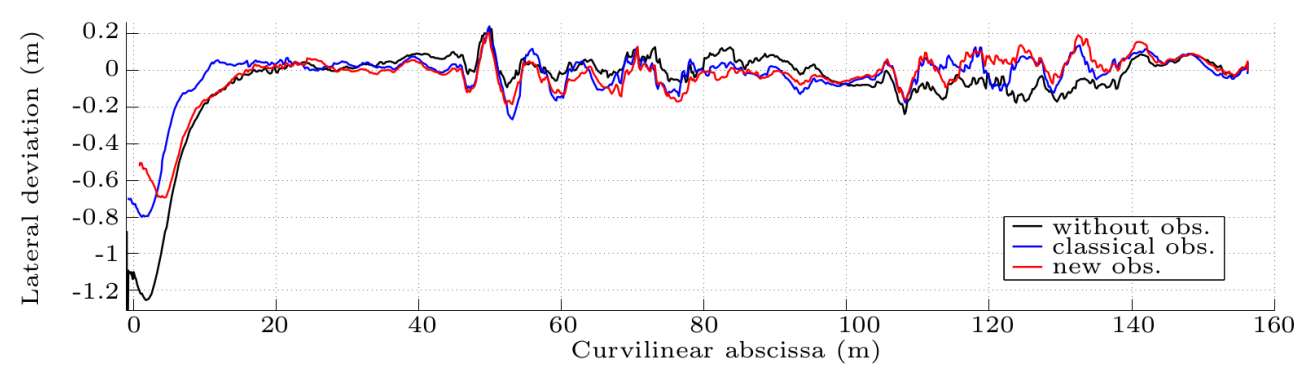

(a) Lateral deviation

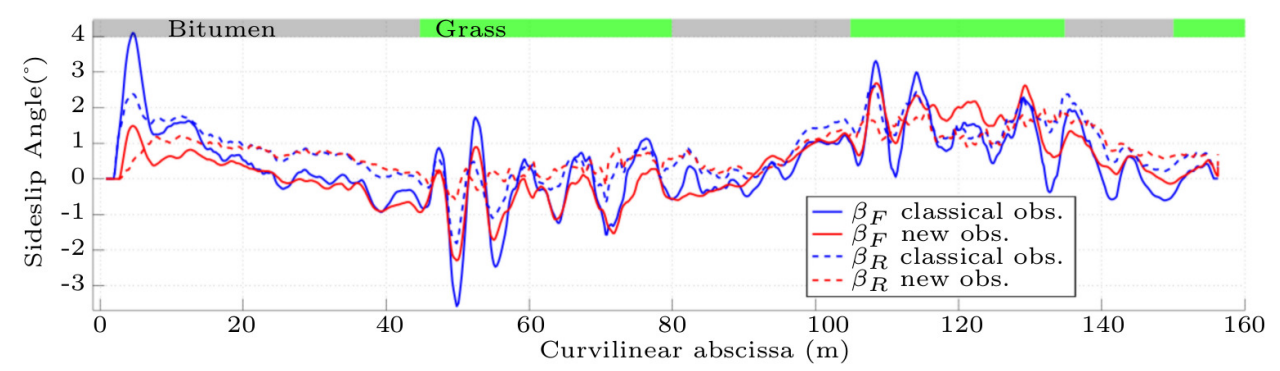

(b) Observed sideslip angles

Figure 9.: Path following and observed sideslip angles at $2 m . s^{-1}$

Figure 9 shows the results obtained at $2 \mathrm{~m} . \mathrm{s}^{-1}$. The lateral deviations recorded during the three tests and the sideslip angles $\left(\beta_{F}\right.$ and $\left.\beta_{R}\right)$ computed with previous and new observers during the third test (i.e. path tracking achieved with the new observer, the sideslip angles computed with the previous one are not used) are presented. These results show that at $2 \mathrm{~m} . \mathrm{s}^{-1}$, path following performances during the first part of the tests are identical in the three cases. However, from curvilinear abscissa $80 \mathrm{~m}$ (which corresponds to the beginning of the second curve), the lateral deviation presents a bias of $0.15 \mathrm{~m}$ during the test without observer. On the contrary, when using adaptive control (sideslip angles are not set to zero), the error stays very close to zero, whatever the observation method used for the estimation of sideslip angles.

The estimated sideslip angles obtained with the previous observer are compared to the ones obtained with the proposed strategy in Figure 9(b). They are quite similar in this test, since the velocity is quite small and the trajectory has no discontinuity. At such a limited speed, the reactivity of the observer is not 
crucial and sliding is quite limited, whatever the grip conditions (it can indeed be seen that sideslip angles hardly exceed $\left.\pm 2^{\circ}\right)$. As a result, the performances of the new observer at low speed $\left(2 \mathrm{~m} \cdot \mathrm{s}^{-1}\right)$ is similar to the performances of the previous one.

The comparison at higher speed $\left(4 \mathrm{~m} \cdot \mathrm{s}^{-1}\right)$ is presented in Figure 10. The three tests are identical to the three previous ones, only the velocity during path tracking has been increased. Figure 10(a) compares the lateral deviations obtained during the tracking in the three configurations : (i) without sliding accounted (in black line), (ii) with sideslip angles observed with the previous method (in blue line) and (iii) using sideslip angles estimated with the proposed observer (in red line). It can be noticed that the proposed observation strategy ensures higher performances. The maximal lateral deviation is indeed about $\pm 0.4 \mathrm{~m}$ when using observer (8). On the contrary, when using the previous observer, overshoots of $\pm 0.8 \mathrm{~m}$ are recorded when the sideslip angles are quickly varying (which is typically the case at curvature transitions, especially at the beginning/end of curves). Nevertheless, during steady state phases (in straight line or when the path curvature is constant), both adaptive strategies ensure a similar satisfactory tracking accuracy. On the contrary, when sliding is neglected, the robot is not able to follow the trajectory during the bends. Important deviations are recorded : $0.5 \mathrm{~m}$ when the robot describes the left bend on asphalt, next the deviation is progressively reduced to $0.35 \mathrm{~m}$ when it continues on grass, and eventually the maximal deviation is equal to $-0.5 \mathrm{~m}$ when it achieves the second curve.

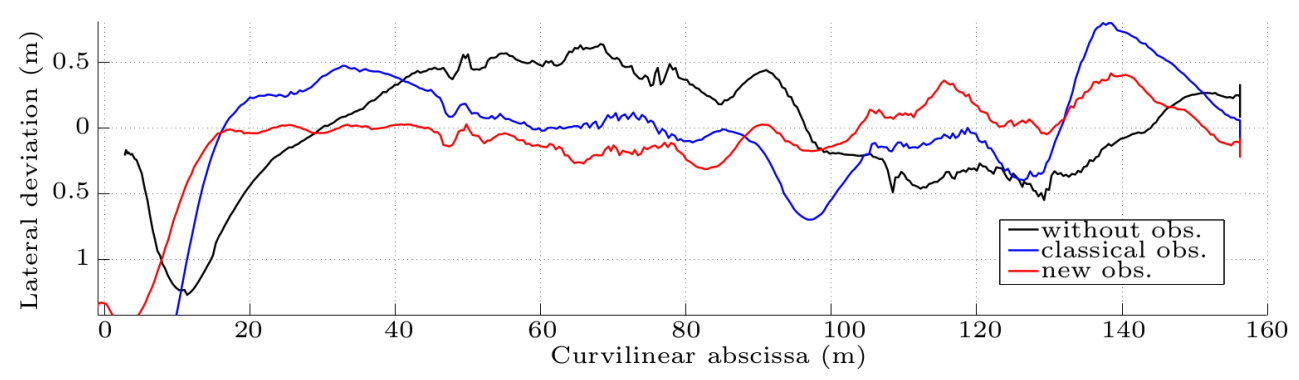

(a) Lateral deviation

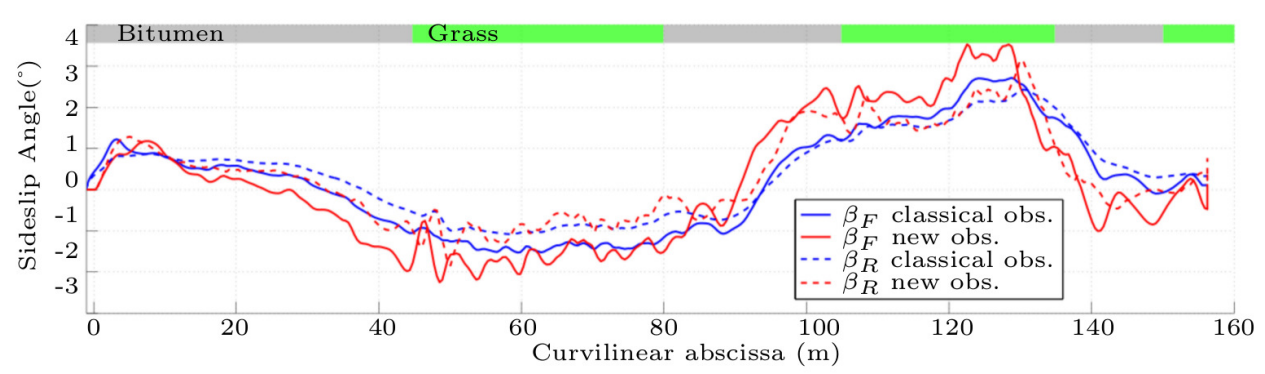

(b) Observed sideslip angles

Figure 10.: Path following and observed sideslip angles at $4 m . s^{-1}$

The improvement in tracking error obtained when using the observer proposed in this paper may be explained by the higher reactivity in the sideslip angle estimation. The observed sideslip angles are compared in Figure 10(b). At each curvature transition (at curvilinear abscissae $35 \mathrm{~m}, 90 \mathrm{~m}, 130 \mathrm{~m}$ and $140 \mathrm{~m}$ ), the red lines (representative of the sideslip angles delivered by the new observer) react faster than the blue ones (representative of the sideslip angles supplied by the previous observer). Of course, during steady state phases, estimated sideslip angles are quite similar (blue and red lines are superimposed). This improved reactivity ensures that the steering control law quickly gets relevant values for $\beta^{F}$ and $\beta^{R}$ at transition. As a result, overshoots at curvature transitions are considerably reduced.

Finally, these tests at $2 \mathrm{~m} \cdot \mathrm{s}^{-1}$ and $4 \mathrm{~m} \cdot \mathrm{s}^{-1}$ show that sideslip angle estimation is not destabilized when 
the nature of the soil changes, since no significant lateral deviation is recorded at ground transitions. However, the influence of soil conditions can nevertheless be noticed in Figure 9(b) and 10(b) : when the robot moves on grass, the observed sideslip angles are indeed a little bit more noisy and reach different values than on asphalt, even if the curvature stays constant. This shows that grip conditions are implicitly accounted by the observers.

To conclude, these results show that at low speed the performances of both observers are identical (when considering a continuous reference path). When the speed is increased, the reactivity of the new observer permits to preserve the accuracy of the path tracking, despite harsh curvature variations and changes in the grip conditions.

\subsubsection{Repeatability}

As illustrated in the previous sections, the proposed adaptive approach is able to deliver high accurate path tracking, whatever the shape of the reference path, the variations in grip conditions and the robot speed. Nevertheless, this level of accuracy has to be repeatable, in the sense that the robot with the proposed control law has to exhibit the same behavior when running in similar conditions. In order to check this point, four successive runs have been performed at a $3 \mathrm{~m} . \mathrm{s}^{-1}$ velocity with the reference trajectory depicted in Figure 8 . Only the departure point may change, the robot does not exactly start at the same position and with the same orientation.

The tracking errors obtained during these four tests are compared in Figure 11. It can be seen that after a settling distance of $20 \mathrm{~m}$, the four tracking errors reach zero and next have a similar behavior. Since the horizon of prediction has been tuned with respect to the highest velocity (set at $0.4 \mathrm{~s}$ for the tests achieved at $4 \mathrm{~m} . \mathrm{s}^{-1}$ ), it is not optimal when the velocity is $3 \mathrm{~m} \cdot \mathrm{s}^{-1}$ and two slight overshoots are recorded at curvilinear abscissae $90 \mathrm{~m}$ and $130 \mathrm{~m}$. Nevertheless, these overshoots permit to highlight the repeatability of the proposed control approach, since it can be noticed that they occur exactly at the same curvilinear abscissae in the four tests.

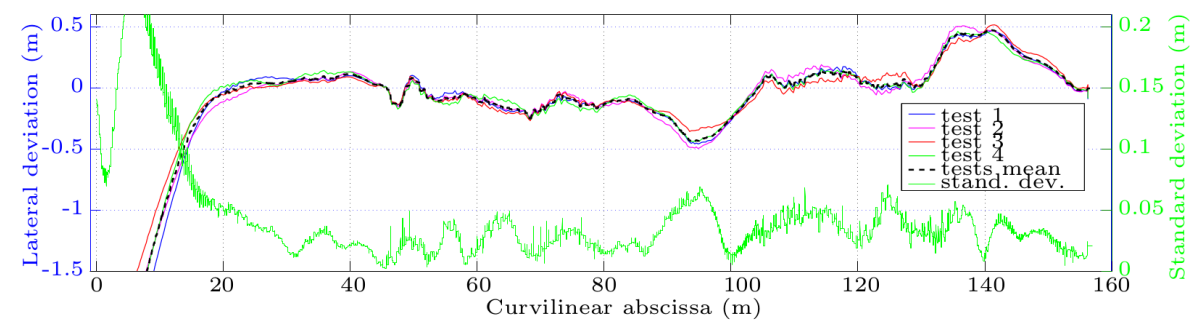

Figure 11.: Lateral deviation and standard deviation

The green line in Figure 11 depicts the standard deviation of the four tracking errors with respect to the curvilinear abscissa. It can be noticed that after the settling distance of $20 \mathrm{~m}$, the standard deviation stays below $0.05 \mathrm{~m}$. This means that almost $70 \%$ of the time, the robot repeats the trajectory within a range of $10 \mathrm{~cm}$, despite the different perturbations and sensor noises.

As mentioned above, the test of repeatability has been performed with a non-optimal horizon of prediction. This has permitted to highlight that the robot always follows the same trajectory, with the same overshoots, when running in the same conditions. This fact also points out the importance of tuning properly the horizon of prediction with respect to the robot speed. The autonomous adaptation of the horizon of prediction is under investigation. In order to discuss further the accuracy of the proposed approach, Figure 11 depicts the tracking error obtained with an horizon of prediction properly set to $0.3 \mathrm{~s}$. It can be noticed that the overshoots are then reduced and the obtained accuracy is close to the repeatability range highlighted above $(10 \mathrm{~cm})$. 


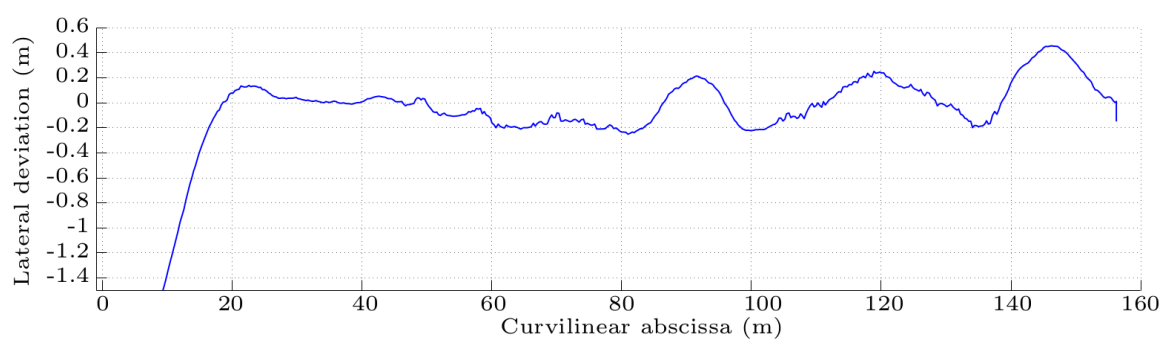

Figure 12.: Lateral deviation at $3 m . s^{-1}$ with an optimized horizon of prediction

Finally, Table 2 and Table 3 present some statistical data, namely the mean and standard deviation of the absolute values of the tracking error recorded either during the first curve (curve to the left with a constant curvature - Table 2) or the second curve (curve to the right with an increasing curvature - Table 3) for different robot's velocities, when neglecting sideslip angles ( $\beta^{F}$ and $\beta^{R}$ are set to zero), when using the previous observer and when using the proposed approach.

\begin{tabular}{|l|l|l|l|l|l|l|}
\hline & \multicolumn{2}{|c|}{ Without sliding accounted } & \multicolumn{2}{c|}{ Previous observer } & \multicolumn{2}{c|}{ Proposed observer } \\
\hline speed & $\operatorname{mean}(|y|)$ & $\operatorname{std}(|y|)$ & $\operatorname{mean}(|y|)$ & $\operatorname{std}(|y|)$ & $\operatorname{mean}(|y|)$ & $\operatorname{std}(|y|)$ \\
\hline $2 m . s^{-1}$ & 0.05 & 0.04 & 0.06 & 0.05 & 0.05 & 0.05 \\
\hline $3 m . s^{-1}$ & 0.38 & 0.13 & 0.11 & 0.09 & 0.10 & 0.05 \\
\hline $4 m . s^{-1}$ & 0.38 & 0.17 & 0.17 & 0.16 & 0.12 & 0.09 \\
\hline
\end{tabular}

Table 2.: Comparison of the properties of the tracking error signal recorded during the first curve

\begin{tabular}{|l|l|l|l|l|l|l|}
\hline & \multicolumn{2}{|c|}{ Without sliding accounted } & \multicolumn{2}{c|}{ Previous observer } & \multicolumn{2}{c|}{ Proposed observer } \\
\hline speed & $\operatorname{mean}(|y|)$ & $\operatorname{std}(|y|)$ & $\operatorname{mean}(|y|)$ & $\operatorname{std}(|y|)$ & $\operatorname{mean}(|y|)$ & $\operatorname{std}(|y|)$ \\
\hline $2 m . s^{-1}$ & 0.08 & 0.05 & 0.06 & 0.03 & 0.05 & 0.03 \\
\hline $3 m . s^{-1}$ & 0.26 & 0.08 & 0.13 & 0.10 & 0.14 & 0.08 \\
\hline $4 m . s^{-1}$ & 0.32 & 0.11 & 0.27 & 0.20 & 0.12 & 0.17 \\
\hline
\end{tabular}

Table 3.: Comparison of the properties of the tracking error signal recorded during the second curve

It can be noticed that at low speed, namely $2 \mathrm{~m} \cdot \mathrm{s}^{-1}$, the benefit of estimating the sideslip angles is questionable, since it does not impact significantly the tracking accuracy : whether the sideslip angles are estimated or not, the mean as well as the standard deviation of the absolute values of the lateral error remain close to zero (around $5 \mathrm{~cm}$ ) in all cases.

The importance of considering adaptive control appears clearly at higher speed $\left(3 \mathrm{~m} \cdot \mathrm{s}^{-1}\right.$ or $\left.4 \mathrm{~m} \cdot \mathrm{s}^{-1}\right)$. The mean and standard deviation are indeed very significant when sliding effects are neglected : the mean error reaches $38 \mathrm{~cm}$ during the first curve (Table 2) and is superior to $26 \mathrm{~cm}$ during the second one (Table 3). Practically, when sideslip angles are neglected, the robot drifts up to $60 \mathrm{~cm}$ from its reference path, as illustrated in Figure 10 or in Figure 4.

On the contrary, tracking accuracy can be preserved if sliding effects are taken into account. At moderate velocity $\left(3 \mathrm{~m} \cdot \mathrm{s}^{-1}\right)$, similar satisfactory performances can be achieved with any of the two observers : the mean error is around $10 \mathrm{~cm}$ in the first curve (Table 2) and around $13 \mathrm{~cm}$ in the second one (Table 3). It can however be noticed that the observer proposed in this paper ensures a lower standard deviation. At higher velocity $\left(4 \mathrm{~m} . \mathrm{s}^{-1}\right)$, the performances of the two observers differ : the previous observer still permits an increase in tracking accuracy with respect to the classical steering law where sliding effects are disregarded, especially when these effects are slowly varying (when the reference path curvature is almost constant, see Table 2), but the performances are largely depreciated with respect to the ones obtained at lower velocities (especially when the reference path curvature is varying, see Table 3 ). In contrast, the 
tracking accuracy obtained with the observer proposed in the paper is unchanged : the mean error is still around $12 \mathrm{~cm}$, the reference path curvature varying or not. As mentioned above, in addition to be sensitive to reference path discontinuities, the previous observer lacks of reactivity, so that the tracking error is depreciated when robot's speed is increased, especially when the reference path curvature is fast varying. The observer proposed in this paper is more reactive, as pointed out above, so that the lateral deviation is rather unaffected by the robot's velocity. This demonstrates the robustness of the proposed observer with respect to speed, terrain conditions and the shape of the trajectory.

\section{Conclusion and perspectives}

In this paper, an observer-based adaptive control strategy has been proposed in order to achieve high accurate path tracking when mobile robots are subject to non-ideal grip conditions. First, two extended kinematic models, where sliding effects are incorporated by means of sideslip angles, have been derived, the first one being expressed in an absolute frame and the second one in a frame attached to the reference path. A first sideslip angle observer designed from the second model, i.e. relying on state variables defined with respect to the reference path, has then been recalled. Next, an original observer has been designed from the first model expressed in an absolute frame. More precisely, sideslip angles have been considered as additional state variables without dynamics, but a proper adaptation law ensures the stability of the observer without any restrictive assumption, contrarily to the previous approach. In particular, since it relies only on the robot absolute motion, it ensures a smoother and more robust estimation with respect to possible reference path discontinuities. Moreover, the proposed estimation strategy reacts faster and permits to increase the robustness with respect to speed variations.

As a result, the estimated sideslip angles and consequently the path tracking accuracy are less sensitive to the noise and to fast modifications of the reference path (that can be misinterpreted as sliding motion). These attractive features have been checked through simulations and experimentations. They are particularly interesting when the reference path is built online from the data received from a moving target ahead, or when multiple trajectories are defined (for obstacle avoidance purpose) without continuous transition from one to the others. Moreover, the proposed approach is independent from the robot velocity, so that the tracking of a target that can possibly stop can be considered (this also opens the way to a practical stabilization of off-road robots from a path tracking approach).

Finally, this proposed path tracking strategy based on extended kinematic models is suitable as long as dynamical effects are negligible. For the robot and the terrain considered here, dynamical effects start influencing the motion beyond $3 \mathrm{~m} \cdot \mathrm{s}^{-1}$, while it is around $2 \mathrm{~m} \cdot \mathrm{s}^{-1}$ for small robots and $7 \mathrm{~m} . \mathrm{s}^{-1}$ for bigger mobiles (such as described in [12]). To address higher velocities, partial dynamical models such as the ones proposed in [17] could be considered to supplement the observer model with an expression for the derivative of the sideslip angles. Additional variables and possible additional sensors may be required in order to preserve the system observability, but this could permit to track possibly discontinuous paths at high speed on natural ground.

\section{Acknowledgment}

This work has received the support of French National Research Agency under the grant number ANR14-CE27-0004 attributed to Adap2E project (adap2e.irstea.fr). It has also been sponsored through the RobotEx Equipment of Excellence (ANR-10-EQPX-44), by the European Union through the program Regional competitiveness and employment 2007-2013 (ERDF Auvergne Region), and by the Auvergne Region. 


\section{Références}

[1] B. d'Andréa-Novel, G. Campion, and G. Bastin. Control of wheeled mobile robots not satisfying ideal velocity constraints : a singular perturbation approach. International Journal of Robust and Nonlinear Control, 5(4) :243$267,1995$.

[2] S. Blackmore, B. Stout, M. Wang, and B. Runov. Robotic agriculture - the future of agricultural mechanisation? In European Conference on Precision Agriculture (ECPA), pages 621-628, Uppsala (Sweden), 2005.

[3] M. Buehler, K Iagnemma, and S. Sanjiv. The 2005 DARPA Grand Challenge : the Great Robot Race, volume 36. Springer Tracts in Advanced Robotics, 2007.

[4] G. Campion, G. Bastin, and B. d'Andréa-Novel. Structural properties and classification of kinematic and dynamic models of wheeled mobile robots. IEEE Transactions on Robotics and Automation, 12(1) :47-62, 1996.

[5] C. Canudas-de Wit, P. Tsiotras, X. Claeys, J. Yi, and R. Horowitz. Friction tire/road modeling, estimation and optimal braking control. In NACO2 Workshop, volume 45, Lund, Sweden, 2001.

[6] C. Cariou, R. Lenain, B. Thuilot, and M. Berducat. Automatic guidance of a four-wheel-steering mobile robot for accurate field operations. Journal of Field Robotics, 26(6-7) :504-518, 2009.

[7] L.M. Corradini, T. Leo, and G. Orlando. Experimental testing of a discrete-time sliding mode controller for trajectory tracking of a wheeled mobile robot in the presence of skidding effects. Journal of Robotic Systems, 19(4) :177-188, 2002.

[8] M. Doumiati, A.C. Victorino, A. Charara, and D. Lechner. Onboard real-time estimation of vehicle lateral tire-road forces and sideslip angle. IEEE/ASME Transactions on Mechatronics, 16(4) :601-614, 2011.

[9] T.D. Gillespie. Fundamentals of vehicle dynamics. Number R-114. Society of Automotive Engineers (SAE), Warrendale (USA), 1992.

[10] J. Guldner and V.I. Utkin. Stabilization of non-holonomic mobile robots using Lyapunov functions for navigation and sliding mode control. In IEEE Conference on Decision and Control (CDC), volume 3, pages 2967-2972, Lake Buena Vista (USA), 1994.

[11] B. Hamner, M. Bergerman, and S. Singh. Autonomous orchard vehicles for specialty crops production. In Annual International Meeting of American Society of Agricultural and Biological Engineers (ASABE), Louisville (USA), 2011.

[12] M. Krid and F. Ben Amar. Design and control of an active anti-roll system for a fast rover. In IEEE/RSJ International Conference on Intelligent RObots and Systems (IROS), pages 274-279, San Francisco (USA), 2011.

[13] Y. Kuwata, J. Teo, G. Fiore, S. Karaman, E. Frazzoli, and J.P. How. Real-time motion planning with applications to autonomous urban driving. IEEE Transactions on Control Systems Technology, 17(5) :1105-1118, 2009.

[14] R. Lenain, B. Thuilot, C. Cariou, and P. Martinet. Rejection of sliding effects in car-like robot control : application to farm vehicle guidance using a single RTK GPS sensor. In IEEE/RSJ International Conference on Intelligent RObots and Systems (IROS), volume 4, pages 3811-3816, Las Vegas (USA), 2003.

[15] R. Lenain, B. Thuilot, C. Cariou, and P. Martinet. High accuracy path tracking for vehicles in presence of sliding : application to farm vehicle automatic guidance for agricultural tasks. Autonomous robots, 21(1) :79-97, 2006.

[16] R. Lenain, B. Thuilot, C. Cariou, and P. Martinet. Adaptive and predictive path tracking control for off-road mobile robots. European Journal of Control, 13(4) :419-439, 2007.

[17] R. Lenain, B. Thuilot, C. Cariou, and P. Martinet. Mixed kinematic and dynamic sideslip angle observer for accurate control of fast off-road mobile robots. Journal of Field Robotics, 27(2) :181-196, 2010.

[18] C. B. Low and D. Wang. Robust path following of car-like WMR in the presence of skidding effects. In IEEE International Conference on Mechatronics (ICM), pages 864-89, Taipei (Taiwan), 2005.

[19] E. Lucet, C. Grand, D. Salle, and P. Bidaud. Stabilization algorithm for a high speed car-like robot achieving steering maneuver. In IEEE International Conference on Robotics and Automation (ICRA), pages 2540-2545, Pasadena (USA), 2008.

[20] A.S. Matveev, M. Hoy, J. Katupitiya, and A. V. Savkin. Nonlinear sliding mode control of an unmanned agricultural tractor in the presence of sliding and control saturation. Robotics and Autonomous Systems, 61(9) :973-987, 2013.

[21] P. Morin and C. Samson. Practical stabilization of a class of nonlinear systems. Application to chain systems and mobile robots. In IEEE Conference on Decision and Control (CDC), volume 3, pages 2989-2994, Sydney (Australia), 2000.

[22] H.B. Pacejka. Tire and vehicle dynamics, $3^{\text {rd }}$ edition. Elsevier, 2012.

[23] C. Samson. Control of chained systems. application to path following and time-varying point stabilization of 
mobile robots. IEEE Transactions on Automatic Control, 40(1) :64-77, 1995.

[24] B. Siciliano and O. Khatib. Handbook of robotics, $2^{\text {nd }}$ edition. Springer Science \& Business Media, 2016.

[25] K. Toussaint, N. Pouliot, and S. Montambault. Transmission line maintenance robots capable of crossing obstacles : state-of-the-art review and challenges ahead. Journal of Field Robotics, 26(5) :477-499, 2009.

[26] D. Wang and C.B. Low. An analysis of wheeled mobile robots in the presence of skidding and slipping : control design perspective. In IEEE International Conference on Robotics and Automation (ICRA), pages 2379-2384, Roma (Italy), 2007.

[27] J. Yi, H. Wang, J. Zhang, D. Song, S. Jayasuriya, and J. Liu. Kinematic modeling and analysis of skid-steered mobile robots with applications to low-cost inertial-measurement-unit-based motion estimation. IEEE Transactions on Robotics, 25(5) :1087-1097, 2009. 